This is a post-peer-review version of an article published in Parkinson and Related Disorders. The final authenticated version is available online at https://doi.org/10.1016/j.parkreldis.2019.10.005

Short communication

\title{
Education level affects dual-task gait after deep brain stimulation in Parkinson's disease
}

\author{
Jennifer Wegrzyk ${ }^{\mathrm{a}, \mathrm{b}, \mathrm{d}, *}$, Stéphane Armand ${ }^{\mathrm{a}, \mathrm{b}}$, Sabina Catalano Chiuvéa ${ }^{\text {, Pierre R. Burkhard }}{ }^{\mathrm{a}}$, \\ Gilles Allali ${ }^{\mathrm{a}, \mathrm{c}}$ \\ ${ }^{a}$ Department of Neurology, Geneva University Hospitals, Geneva, Switzerland \\ ${ }^{\mathrm{b}}$ Willy Taillard Laboratory of Kinesiology, Geneva University Hospitals and University of Geneva, Geneva, Switzerland \\ ${ }^{\mathrm{c}}$ Department of Neurology, Albert Einstein College of Medicine, Yeshiva University, USA \\ ${ }^{\mathrm{d}}$ School of Health Sciences (HESAV), University of Applied Sciences and Arts Western Switzerland (HES-SO), Lausanne, Switzerland
}

\section{A R T I C L E I N F O}

\section{Keywords:}

Gait

Parkinson's disease

Deep brain stimulation

Dual-task

Cognitive reserve

\begin{abstract}
A B S T R A C T
Introduction: High cognitive reserve is associated with milder cognitive and motor deficits in Parkinson's disease (PD). We investigated whether educational status (as a proxy for cognitive reserve) could modulate dual-task (DT) related gait changes after subthalamic nucleus deep brain stimulation (STN-DBS) in PD.

Methods: DT-related gait changes were assessed in 34 PD patients (age: $60.5 \pm 8.7 ; \%$ female: 44\%), before and one year after STN-DBS. Based on walking speed change after DBS, patients were classified into responders (improvement) and non-responders (deterioration) using automated k-means clustering for four DT (i.e. forward and backward counting; semantic and phonemic fluency).

Results: Patients with high education level improved DT gait performance compared to lower educated patients $(\mathrm{p}=0.03)$. Baseline cognitive performance, disease progression and stimulation efficiency were similar between groups (i.e. responders versus non-responders). Logistic regression showed an association between responders and high level of education for verbal fluency (semantic/phonemic fluency, beta $=3.9 / 3.4, p=0.03$ ). No significant changes for any gait parameter were found using all-group analyses.

Conclusion: Education level is associated with DT-related gait changes in PD one year post-DBS. Subgroup analyses should be considered for highly variable gait outcomes after STN-DBS. With regard to the predominance of motor-cognitive DT performance in everyday life, a high CR could be considered as a favourable inclusion criterion for future DBS candidates.
\end{abstract}

\section{Introduction}

Education, occupational attainment and leisure activities contribute to cognitive reserve (CR) thus maintaining higher brain functions over time [1]. CR accounts for differences between individuals in susceptibility to age- or pathology-related brain changes and has been largely studied in Alzheimer's disease [1]. In Parkinson's disease (PD), high CR is associated with both milder cognitive and motor deficits [2]. Only one recent study addressed the relationship between CR and motor impairment in PD patients that underwent deep brain stimulation (DBS) showing that higher education was inversely related to cardinal motor disabilities two years after DBS [3].

The impact of CR on dual-tasks (DT) has not yet been investigated in PD. The DT-gait paradigm involves the simultaneous realization of a cognitive task while walking; representing an accepted design to evaluate motor-cognitive impairments that reflect attention demanding, everyday life conditions in which the risk of falling is increased [4]. Two studies investigated the effects of STN-DBS on DT-related gait only post-DBS during 'on' and 'off' stimulation conditions and showed conflicting results $[5,6]$. A possible reason for these inconsistent findings is that these studies performed all-group analyses thereby not sufficiently considering the between-patient variability of gait outcome after DBS [7]. Consequently, the present study aims at identifying and comparing subgroups of patients with successful and less successful outcome (i.e. responder versus non-responders).

To date, it is unknown if PD patients with higher level of education are more resilient to DT-related gait impairment after DBS. Establishing the impact of $\mathrm{CR}$ on DT-related gait changes may improve selection

\footnotetext{
* Corresponding author. School of Health Sciences (HESAV), University of Applied Sciences and Arts Western Switzerland (HES-SO), Avenue de Beaumont 21, 1011 Lausanne, Switzerland

E-mail address: jennifer.masset@hesav.ch (J. Wegrzyk).
} 
Table 1

(A) Baseline characteristics and (B) Pre vs. post-DBS changes between Responders and non-Responders $(\mathrm{n}=34)$. UPDRS: Unified Parkinson Disease Rating Scale (UPDRS) III.

\begin{tabular}{|c|c|c|c|}
\hline & Responders $(n=18)$ & Non-Responders $(\mathrm{n}=16)$ & p-value \\
\hline Age at surgery (yrs) & $59.39 \pm 10.36$ & $61.75 \pm 6.55$ & 0.670 \\
\hline Gender ( $\%$ age female) & 39.9 & 50.0 & 0.515 \\
\hline Disease duration (yrs) & $11.38 \pm 4.19$ & $10.69 \pm 3.72$ & 0.611 \\
\hline \multicolumn{4}{|l|}{ Level of education (1-3) } \\
\hline 1. Primary level & 1 & 7 & $0.030 *$ \\
\hline 2. Secondary level & 8 & 4 & \\
\hline 3. High level & 9 & 5 & \\
\hline Levodopa Equivalent Daily Dose & $1584 \pm 513$ & $1383 \pm 387$ & 0.222 \\
\hline Levodopa response (UPDRS III, \%) & $64.82 \pm 21.18$ & $62.19 \pm 15.11$ & 0.685 \\
\hline UPDRS III (on med) & $13.08 \pm 10.14$ & $17.28 \pm 17.04$ & 0.422 \\
\hline Apathy (Starkstein) & $9.39 \pm 3.62$ & $9.69 \pm 3.30$ & 0.804 \\
\hline Mattis Total (/144) & $138.56 \pm 6.12$ & $137.87 \pm 5.23$ & 0.484 \\
\hline Attention (/37) & $36.44 \pm 0.92$ & $36.06 \pm 0.93$ & 0.187 \\
\hline Initiation (/37) & $34.83 \pm 2.90$ & $35.00 \pm 2.83$ & 1.000 \\
\hline Construction (/6) & $6.00 \pm 0.00$ & $5.94 \pm 0.25$ & 0.772 \\
\hline Conceptualization (/39) & $36.94 \pm 2.31$ & $37.37 \pm 1.71$ & 0.746 \\
\hline Memory $(/ 25)$ & $24.33 \pm 0.97$ & $23.50 \pm 1.75$ & 0.224 \\
\hline \multicolumn{4}{|l|}{ Gait Speed (Cognitive Scores) } \\
\hline Single task $(\mathrm{m} / \mathrm{s})$ & $1.20 \pm 0.23$ & $1.17 \pm 0.30$ & 0.656 \\
\hline Forward Counting $(\mathrm{m} / \mathrm{s})(\mathrm{score} / \mathrm{s})$ & $1.17 \pm 0.25(1.60 \pm 0.99)$ & $1.14 \pm 0.21(1.88 \pm 0.90)$ & $0.708(0.06)$ \\
\hline Backward Counting $(\mathrm{m} / \mathrm{s}){ }^{\#}$ (score $\left./ \mathrm{s}\right)$ & $1.07 \pm 0.22(1.69 \pm 0.88)$ & $1.06 \pm 0.24(1.42 \pm 0.84)$ & $0.886(0.22)$ \\
\hline Semantic Fluency $(\mathrm{m} / \mathrm{s}){ }^{\#}($ score $/ \mathrm{s})$ & $0.91 \pm 0.23(0.99 \pm 0.38)$ & $0.98 \pm 0.24(0.91 \pm 0.47)$ & $0.405(0.70)$ \\
\hline Phonemic Fluency $(\mathrm{m} / \mathrm{s}){ }^{\#}$ (score $\left./ \mathrm{s}\right)$ & $0.83 \pm 0.28(0.98 \pm 0.94)$ & $0.92 \pm 0.23(0.78 \pm 0.55)$ & $0.339(0.98)$ \\
\hline \multicolumn{4}{|l|}{ UPDRS } \\
\hline Med on & $11.64 \pm 8.79$ & $15.06 \pm 9.24$ & 0.284 \\
\hline Med off & $36.68 \pm 12.86$ & $40.60 \pm 15.63$ & 0.469 \\
\hline \multicolumn{4}{|l|}{ B) } \\
\hline & Responders $(n=18)$ & Non-Responders $(n=16)$ & p-value \\
\hline \multicolumn{4}{|l|}{$\Delta$ Clinical values $(\%)$} \\
\hline Levodopa Equivalent Daily Dose & $-70.20 \pm 21.09$ & $-64.41 \pm 22.81$ & 0.322 \\
\hline Mattis (total) & $1.28 \pm 2.43$ & $-1.51 \pm 3.66$ & 0.019 \\
\hline UPDRS III (best on) & $-12.36 \pm 64.88$ & $-5.56 \pm 79.56$ & 0.798 \\
\hline \multicolumn{4}{|l|}{$\Delta$ Gait Speed (m/s) (\%) } \\
\hline Single Task & $5.76 \pm 15.83$ & $-7.49 \pm 18.49$ & $<0.05$ \\
\hline Forward counting & $18.34 \pm 30.75$ & $-14.90 \pm 10.36$ & $<0.0005$ \\
\hline Backward counting & $18.70 \pm 14.60$ & $-16.79 \pm 13.53$ & $<0.0005$ \\
\hline Semantic fluency & $27.62 \pm 24.19$ & $-19.60 \pm 17.93$ & $<0.0005$ \\
\hline Phonemic fluency & $40.19 \pm 54.97$ & $-19.83 \pm 17.57$ & $<0.0005$ \\
\hline \multicolumn{4}{|l|}{ UPDRS III ratio } \\
\hline Stimulation efficiency ((stim on/med off)/med off) & $0.74 \pm 0.48$ & $0.53 \pm 0.69$ & 0.302 \\
\hline Disease progression ((stim off/med off)/med off) & $0.017 \pm 0.485$ & $-0.287 \pm 0.459$ & 0.135 \\
\hline
\end{tabular}

Data presented as mean $\pm \mathrm{SD}$. ${ }^{\#}$ : walking speed significantly different than single task (paired $t$-test, $\mathrm{p}<0.005$ ).

criteria for DBS inclusion. Based on recent publications stating the relevance of CR in PD [2], we hypothesized that a higher level of education would positively affect DT-performance one year post-DBS.

\section{Methods}

\subsection{Subjects}

A total of 34 consecutive PD patients (age: $60.5 \pm 8.7 ; \%$ female: 44) were tested $2-4$ months ( $89 \pm 67$ days) before and one year (370 \pm 66 days) after bilateral STN-DBS surgery at the Geneva University Hospitals from 2011 to 2016 . All subjects met the inclusion criteria to undergo STN-DBS and did not rely on any walking aid neither pre nor post-surgery. Electrode implementation and modulation were performed according to clinical routine. The study was approved by the local ethical committee and patients provided informed written consent.

\subsection{Clinical and gait testing}

Clinical and gait testing were performed under best medical treatment (i.e. drug-on condition pre-DBS vs. drug-on and stim-on condition post-DBS) to better reflect the patients' functional abilities in daily life. Motor and levodopa response were assessed via the Unified Parkinson Disease Rating Scale (UPDRS) III and cognitive functioning using the Mattis Dementia Rating Scale (MDRS-2). Level of education was expressed as categorical values ranging from 1 to 3 ( 1 = Primary Level, i.e. compulsory schooling $\leq 9$ years, $2=$ Secondary Level, i.e. apprenticeship 9-12 years, $3=$ High Level, i.e. baccalaureate/university studies $>12$ years).

Gait assessment was performed at comfortable speed in four DTconditions: forward counting (from 1), backward counting (from 50), 
semantic fluency (enumerating animal names) and phonemic fluency (enumerating words with initial letter "p"). For each participant, the order of DT was randomized and kept constant for the pre and post-DBS testing. Each cognitive task was subsequently performed in sitting position for which subjects were given the same amount of time as recorded for the dual-task. The main outcome was "walking speed $(\mathrm{m} / \mathrm{s})$ " that reflects performance of activities of daily living, risk of falling, hospitalization and quality of life [8]. All gait parameters were assessed with a 12-camera optoelectronic system (Oqus7+, Qualisys) synchronised with a pressure sensitive walkway (GAITRite, CIR Systems Inc.) and computed using Matlab (Mathworks).

\subsection{Data analyses}

Data analyses were conducted using $\mathrm{R}$ software (2.15.3). $\mathrm{P}$ values $\leq 0.05$ were considered to indicate statistical significance (two tailed).

Automated, non-hierarchical k-means clustering analysis [9] was performed to objectively differentiate patients that improved post-DBS gait (responders) from those that worsened (non-responders). The clustering was based on the walking speed $(\mathrm{m} / \mathrm{s})$ delta values (post-pre/ pre DBS) of all four DT per patient. Both subgroups were then compared using $\chi^{2}$ (categorical variables); independent $t$-test and Mann-Whitney$\mathrm{U}$ test (continuous variables) according to their distribution (ShapiroWilk). In order to explain changes in walking speed as product of cadence and step length, the latter two factors were analyzed post-hoc for responders and non-responders based on the most significant dual-task.

Univariate and multivariate (adjusted for age and gender) logistic regressions subsequently assessed the association between group belonging (dependent variable) and education level (independent variable). Pre vs. post-DBS changes (based on a delta-score in \%) were assessed for cognitive function (Mattis total and subscores) and spatiotemporal gait parameters (including walking speed, cadence, step length and step width) for all-group instead of cluster-based subgroup data using paired $t$-test and Wilcoxon signed-rank test respectively.

\section{Results}

Eighteen responders and sixteen non-responders were identified using k-means clustering (group characteristics presented in Table 1 for (A) baseline characteristics and (B) delta (pre/post DBS) values).

Level of education was significantly higher in responders compared to non-responder. Comparisons of delta values for each cognitive task assessed in sitting position were not significant between groups. Based on the UPDRS III, no differences with regards to disease progression (i.e. ratio of conditions "stim off/med off" (post) vs. "med off" (pre)) and stimulation efficiency (i.e. conditions "stim on/med off" (post) vs. "med off" (pre)) were found. Also, levodopa equivalent daily dose (LEDD) was not different between groups, neither pre $(p=0.22)$, nor post-surgery $(p=0.59)$. Average walking speed change is plotted per education level (Fig. 1 A) as well as for each DT and subgroup (Fig. 1 B). Responders increased walking speed mainly due to increased cadence (pre: $89 \pm 16$ steps/min vs. post: $104 \pm 12.40$ steps/min, $\mathrm{p}<0.0005)$ whereas non-responders decreased walking speed due to shortened step length (pre: $0.59 \pm 0.12 \mathrm{~m}$ vs. post $0.50 \pm 0.09 \mathrm{~m}$, $\mathrm{p}<0.01$ ).

Logistic regression showed an association between responder/nonresponder and education level (II/III) for all combined DT ( $\beta=3.5 / 3.8$, $p=0.04 / 0.04$ ) and for verbal fluency (semantic fluency: $\beta=3.7 / 3.9$, $\mathrm{p}=0.02 / 0.03$; phonemic fluency: $\beta=2.6 / 3.4, \mathrm{p}=0.06 / 0.03$ ) after adjustment for age, gender and Mattis baseline score (Supplementary Table S1A). Linear regression independent of grouping (using delta gait speed as a continuous measure and as a dependent variable) was significant for all dual-tasks between education level I and education level III after adjustment for age, gender and Mattis baseline score (Supplementary Table S1B) thereby confirming the association between group belonging and education level (S1A).

There was a small but statistically significant improvement of cognitive performance in the responder group (Mattis total score $=$ preDBS: $138.6 \pm 6.1$ vs. post-DBS: $140.3 \pm 4.4 ; \mathrm{p}=0.032$; subscore "conceptualization" = pre-DBS: $36.9 \pm 2.3$ vs. post-DBS: $38.4 \pm 0.9$; $\mathrm{p}=0.006)$. Mattis total score was slightly improved when comparing the pre-post DBS delta score (responders: $+1.28 \pm 2.43 \%$ vs. nonresponders: $-1.51 \pm 3.66 \% ; \mathrm{p}=0.019$ ).

No significant changes were found for all-group spatiotemporal gait data pre vs. post DBS for any DT: walking speed (1.01 \pm 0.21 vs. $1.02 \pm 0.27 \mathrm{~m} / \mathrm{s})$; step length $(0.62 \pm 0.10$ vs. $0.60 \pm 0.11 \mathrm{~m})$; cadence $(97.17 \pm 11.0$ vs. $101.54 \pm 15.32$ steps $/ \mathrm{min})$; step width $(0.08 \pm 0.03$ vs. $0.08 \pm 0.02 \mathrm{~m})$ (averaged data for all DT). LEDD was significantly reduced post-surgery $(1495 \pm 501$ pre vs. $467 \pm 299 \mathrm{mg}$ post, $\mathrm{p}<0.001$ ).

\section{Discussion}

Using the DT-gait paradigm, we assessed the effect of education level on cognitive-motor interference pre and post STN-DBS in PD. Education level, as a relevant proxy for cognitive reserve (CR) [2], was associated with DT-related gait changes one year after STN-DBS.

The findings support the CR theory for complex gait performance in PD. White matter integrity and/or reduced $\beta$-amyloid accumulation might be favoured by higher education status [10], thus explaining its "protective" effect on DT-gait speed that reflects higher order cognitive control [4]. Baseline cognitive scores were similar between both groups and only responders improved cognitive function post-DBS. Patients with high CR might present less vulnerability to executive functions associated with gait control after DBS. Future studies on structural and functional frontal brain networks are needed to explore the brain mechanisms modulated by education status.

Education level had the most positive impact on DT verbal (i.e. semantic and phonemic) fluency, i.e. word-finding facility while walking. Interestingly, verbal fluency was reported to decline following STN-DBS in PD without consistent decline in executive domains and memory [11]. Our results suggest that patient-inherent characteristics (i.e. CR) are partly responsible for post-DBS worsening of verbal fluency. Preserved fronto-temporal circuits could account for improved lexico-semantic performance in higher educated patients post-DBS [12].

Logistic regression best distinguished secondary and high education (level 2-3) from primary education (level 1) suggesting that any education superior to obligatory schooling positively affects DT-related gait after DBS in PD. Future studies should include larger samples in order to account for more differentiated levels of education. In addition, other dimensions of CR, such as occupation and premorbid estimated intelligence, as well as clinical gait-related outcomes, such as history of falls, should be considered in future research to strengthen our findings.

No pre vs. post-DBS changes were observed for any gait parameter when data of all patients (i.e. combining responders and non-responders) were averaged. The findings are consistent with previous studies on PD performed before and after STN-DBS in drug-on conditions for single-task and DT-gait paradigms [6] though improvements have been stated by others [5]. These inconsistencies may result from high inter-patient variability in gait outcomes previously reported postDBS [7], when expressed as all-group effects. Alternatively, automated clustering proves as an appropriate approach to extract discriminative information (based on several outcome variables) for certain patient subgroups.

To conclude, educational status affects DT-related gait changes one year post-DBS in PD. Subgroup analyses should be considered for highly variable gait outcome after surgery. With regard to the predominance of motor-cognitive DT performance in everyday life, a high CR could be considered as a favourable inclusion criterion for future DBS candidates. 
A

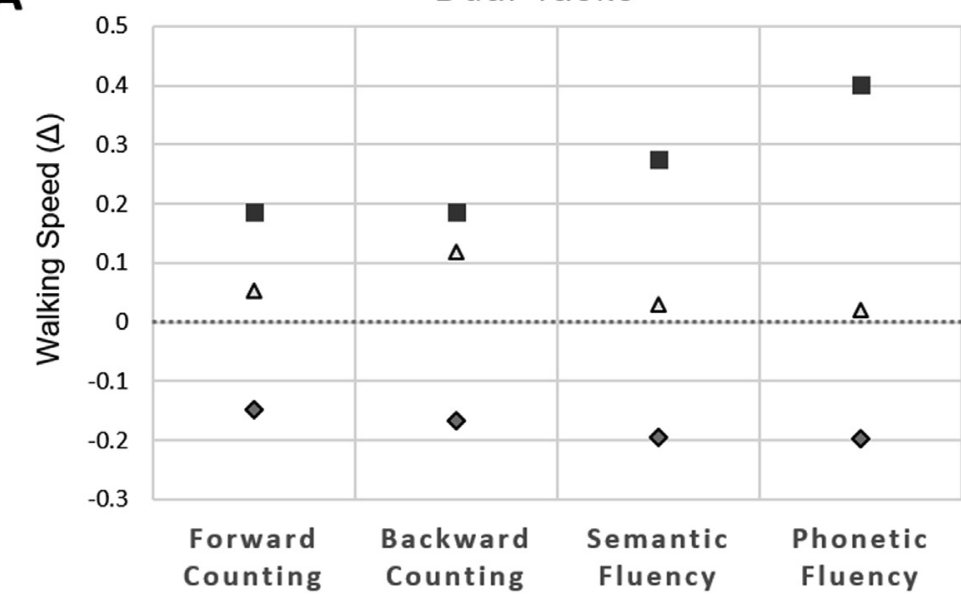

B

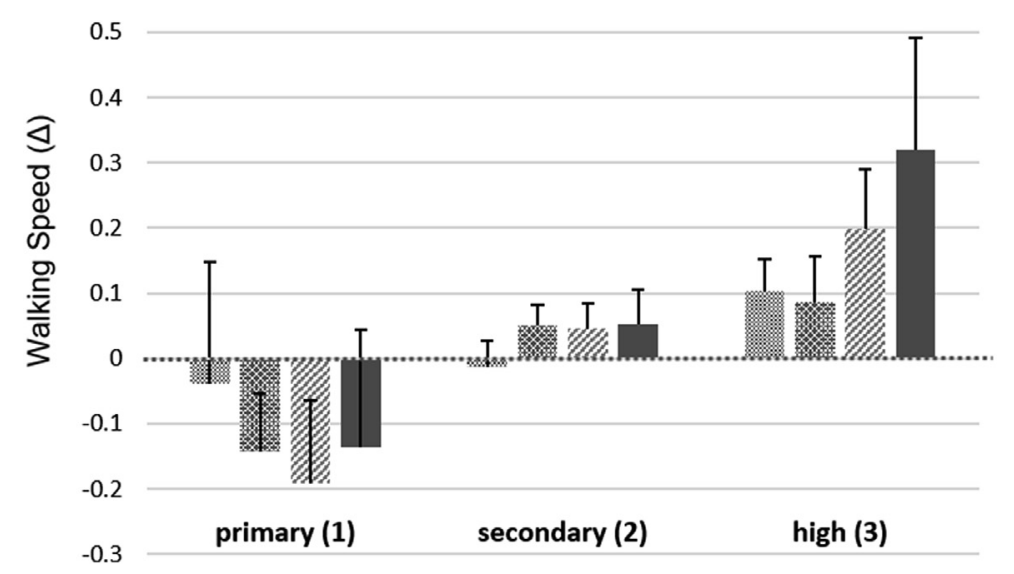

$\diamond$ Non-Responders

- Responders

$\Delta$ Average
Forward Counting

: Backward Counting

\& Semantic Fluency

- Phonetic Fluency

Fig. 1. Walking speed changes (pre vs. post-DBS). (A) between responders and non-responders for each dual-task based on k-means clustering, (B) per education level (1-3) for each dual-task.

\section{Funding sources}

There has been no funding of the study.

\section{Acknowledgements}

We cordially thank all participants who took part in this study.

\section{Appendix A. Supplementary data}

Supplementary data to this article can be found online at https:// doi.org/10.1016/j.parkreldis.2019.10.005.

\section{References}

[1] Y. Stern, Cognitive reserve in ageing and Alzheimer's disease, Lancet Neurol. 11 (11) (2012) 1006-1012.

[2] J.V. Hindle, A. Martyr, L. Clare, Cognitive reserve in Parkinson's disease: a systematic review and meta-analysis, Park. Relat. Disord. 20 (1) (2014) 1-7.

[3] J. Blume, E. Rothenfusser, J. Schlaier, U. Bogdahn, M. Lange, Educational attainment and motor burden in advanced Parkinson's disease - the emerging role of education in motor reserve, J. Neurol. Sci. 381 (2017) 141-143.

[4] V.E. Kelly, A.J. Eusterbrock, A. Shumway-Cook, A review of dual-task walking deficits in people with Parkinson's disease: motor and cognitive contributions, mechanisms, and clinical implications, 2012, Parkinsons Dis (2012) 918-719.

[5] M.E. McNeely, G.M. Earhart, Medication and subthalamic nucleus deep brain stimulation similarly improve balance and complex gait in Parkinson disease, Park. Relat. Disord. 19 (1) (2013) 86-91.

[6] E. Seri-Fainshtat, Z. Israel, A. Weiss, J.M. Hausdorff, Impact of sub-thalamic nucleus deep brain stimulation on dual tasking gait in Parkinson's disease, J. NeuroEng. Rehabil. 10 (2013) 38.

[7] M. Ferrarin, M. Rizzone, B. Bergamasco, M. Lanotte, M. Recalcati, A. Pedotti, L. Lopiano, Effects of bilateral subthalamic stimulation on gait kinematics and kinetics in Parkinson's disease, Exp. Brain Res. 160 (4) (2005) 517-527.

[8] J.A. Roper, N. Kang, J. Ben, J.H. Cauraugh, M.S. Okun, C.J. Hass, Deep brain stimulation improves gait velocity in Parkinson's disease: a systematic review and meta-analysis, J. Neurol. 263 (6) (2016) 1195-1203.

[9] S.M. van Rooden, W.J. Heiser, J.N. Kok, D. Verbaan, J.J. van Hilten, J. Marinus, The identification of Parkinson's disease subtypes using cluster analysis: a systematic review, Mov. Disord. 25 (8) (2010) 969-978.

[10] C. Lucero, M.C. Campbell, H. Flores, B. Maiti, J.S. Perlmutter, E.R. Foster, Cognitive reserve and beta-amyloid pathology in Parkinson disease, Park. Relat. Disord. 21 (8) (2015) 899-904.

[11] H.L. Combs, B.S. Folley, D.T. Berry, S.C. Segerstrom, D.Y. Han, A.J. AndersonMooney, B.D. Walls, C. van Horne, Cognition and depression following deep brain stimulation of the subthalamic nucleus and globus pallidus pars internus in Parkinson's disease: a meta-analysis, Neuropsychol. Rev. 25 (4) (2015) 439-454.

[12] J.V. Baldo, S. Schwartz, D. Wilkins, N.F. Dronkers, Role of frontal versus temporal cortex in verbal fluency as revealed by voxel-based lesion symptom mapping, J. Int. Neuropsychol. Soc. 12 (6) (2006) 896-900. 